\title{
An Optimal Bayesian method for point analysis in discrete time
}

\author{
Fernando Lewis, Geraldo Sclavi, John Copeland, Neil Davidson, and David Anderson \\ Computer Science Department, Upper Lake College
}

\begin{abstract}
In this work we consider time series with a finite number of discrete point changes. We assume that the data in each segment follows a different probability density functions (pdf). We focus on the case where the data in all segments are modeled by Gaussian probability density functions with different means, variances and correlation lengths. We put a prior law on the change point instances (Poisson process) as well as on these different parameters(conjugate priors) and give the expression of the posterior probality distributions of these change points. The computations are done by using an appropriate Markov Chain Monte Carlo (MCMC) technique.

The problem as we stated can also be considered as an unsupervised classification and/or segmentation of the time serie. This analogy gives us the possibility to propose alternative modeling and computation of change points, which are more appropriate for multivariate signals, for example in image processing.
\end{abstract}

key words: Bayesian change-points estimation, classification and segmentation.

\section{INTRODUCTION}

Figure 1 shows typical change point problems we consider in this work. Note that, very often people consider problems in which there is only one change point. ${ }^{1}$ Here we propose to consider more general problems with any number of change points. However, very often the change point analysis problems need online or real time detection algorithms, ${ }^{2-5}, ?$ while here, we focus only on off line methods where we assume that we have gathered all the data and we want to analyse it to detect change points who have been occured during the observation time. Also, even if we consider here change point estimation of 1-D time series, we can extend the proposed method to multivariate data, for example the images where the change point problems become equivalent to segmentation. One more point to position this work is that, very often the models used in change point problems assume to know perfectly the model of the signal in each segment, i.e., a linear or nonlinear regression model, ${ }^{5,6,8-10}$ while here, we use a probabilistic model for the signals in each segment which gives probably more generality and applicability when we do not know perfectly those models.

More specifically, we model the time series by a hierarchical Gauss-Markov modeling with hidden varaibles which are themselves modeled by a Markov model. Though, in each segment

${ }^{*}$ Correspondence: E-mail: djafari@lss.supelec.fr 


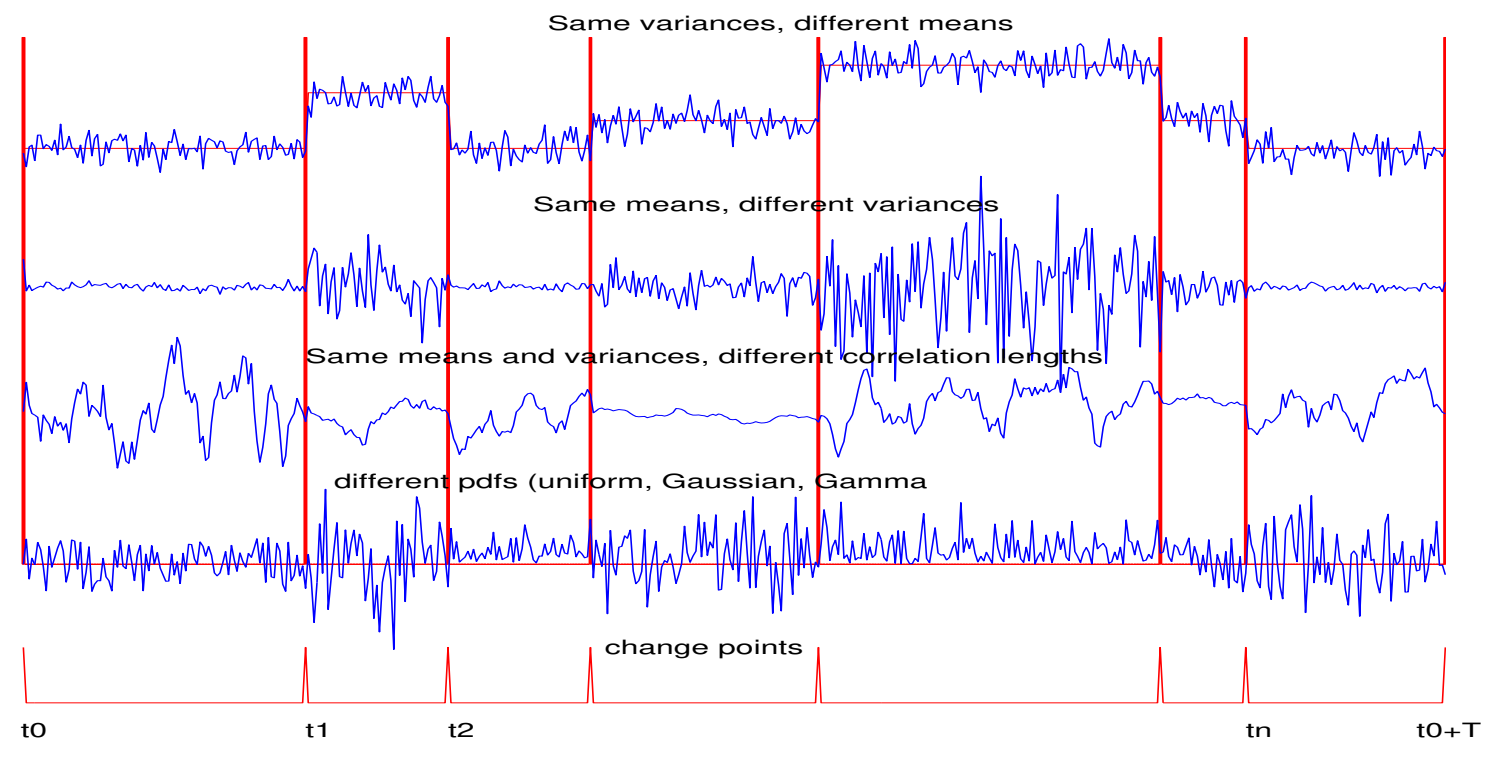

Figure 1. Change point problems description: In the first row, only mean values of the different segments are different. In the second row, only variances are changed. In the third row only the correlation strengths are changed. In the fifth row, the whole nature shape of their probability distribution have been changed. The last row show the change points $t_{n}$.

which corresponds to a particular value of the hidden variable, the time series is assumed to be modeled by a stationnary Gauss-Markov model. However, we choosed a simple parametric model defined only with three parameters of mean $\mu$, variance $\sigma^{2}=1 / \tau$ and a parameter $\rho$ measuring the local correlation strength of the neighboring samples.

The choice of the hidden variable is also important. We have studied three different modeling: i) change point time instants $t_{n}$, ii) classification labels $z_{n}$ or iii) a Bernouilli variable $q_{n}$ which is always equal to zero except when a change point occurs.

The rest of the paper is organized as follows: In the next section we introduce the notations and fixe the objectives of the paper. In section 3 we consider the model with explicite change point times as the hidden variables and propose particular modeling for them and an MCMC algorithm to compute their a posteriori probabilities. In sections 4 and 5 we consider the two other aformentionned models. Finally, we show some simulation results and present our conclusions and perspectives. 


\section{NOTATIONS AND MODELING}

We note by $\boldsymbol{x}=\left[x\left(t_{0}\right), \cdots, x\left(t_{0}+T\right)\right]^{\prime}$ the vector containing the data observed from time $t_{0}$ to $t_{0}+T$. We note by $\boldsymbol{t}=\left[t_{1}, \cdots, t_{N}\right]^{\prime}$ the unknown change points and note $\boldsymbol{x}=\left[\boldsymbol{x}_{0}, \boldsymbol{x}_{1}, \cdots, \boldsymbol{x}_{N}\right]^{\prime}$ where $\boldsymbol{x}_{n}=\left[x\left(t_{n}\right), x\left(t_{n}+1\right), \cdots, x\left(t_{n+1}\right)\right]^{\prime}, \quad n=0, \cdots, N$ represent the data samples in each segment. In the following we will have $t_{N+1}=T$.

We model the data $\boldsymbol{x}_{n}=\left[x\left(t_{n}\right), x\left(t_{n}+1\right), \cdots, x\left(t_{n+1}\right)\right]^{\prime}, \quad n=0, \cdots, N$ in each segment by a Gauss-Markov chain:

$$
\begin{aligned}
p\left(x\left(t_{n}\right)\right)= & \mathcal{N}\left(\mu_{n}, \sigma_{n}^{2}\right) \\
p\left(x\left(t_{n}+l\right) \mid x\left(t_{n}+l-1\right)\right)= & \mathcal{N}\left(\rho_{n} x\left(t_{n}+l-1\right)+\left(1-\rho_{n}\right) \mu_{n}, \sigma_{n}^{2}\left(1-\rho_{n}^{2}\right)\right), \quad l=1, \cdots, l_{n}-1 \\
& l_{n}=t_{n+1}-t_{n}+1=\operatorname{dim}\left[\boldsymbol{x}_{n}\right]
\end{aligned}
$$

Then we have

$$
\begin{aligned}
p\left(\boldsymbol{x}_{n}\right)= & p\left(x\left(t_{n}\right)\right) \prod_{l=1}^{l_{n}} p\left(x\left(t_{n}+l\right) \mid x\left(t_{n}+l-1\right)\right) \\
p\left(\boldsymbol{x}_{n}\right) \propto & \exp \left\{-\frac{1}{2 \sigma_{n}^{2}}\left(x\left(t_{n}\right)-\mu_{n}\right)^{2}\right\} \\
& \exp \left\{-\frac{1}{2\left(\sigma_{n}^{2}\left(1-\rho_{n}^{2}\right)\right)} \sum_{l=1}^{l_{n}}\left[x\left(t_{n}+l\right)-\rho_{n} x\left(t_{n}+l-1\right)-\left(1-\rho_{n}\right) \mu_{n}\right]^{2}\right\} \\
p\left(\boldsymbol{x}_{n}\right)= & \mathcal{N}\left(\mu_{n} \mathbf{1}, \Sigma_{n}\right) \text { with } \boldsymbol{\Sigma}_{n}=\sigma_{n}^{2} \operatorname{Toeplitz}\left(\left[1, \rho_{n}, \rho_{n}^{2}, \cdots, \rho_{n}^{l_{n}}\right]\right)
\end{aligned}
$$

Noting by $\boldsymbol{t}=\left[t_{1}, \cdots, t_{N}\right]$ the vector of the change points and assuming that the samples from any two segments are independent, we can write:

$$
p(\boldsymbol{x} \mid \boldsymbol{t}, \boldsymbol{\theta}, N)=\prod_{n=0}^{N} \mathcal{N}\left(\mu_{n} \mathbf{1}, \boldsymbol{\Sigma}_{n}\right)=\left(\prod_{n=0}^{N} \frac{\left|\boldsymbol{\Sigma}_{n}\right|^{-1 / 2}}{(2 \pi)^{\left(l_{n} / 2\right)}}\right) \exp \left\{-\frac{1}{2} \sum_{n=0}^{N}\left(\boldsymbol{x}_{n}-\mu_{n} \mathbf{1}\right)^{\prime} \boldsymbol{\Sigma}_{n}^{-1}\left(\boldsymbol{x}_{n}-\mu_{n} \mathbf{1}\right)\right\}
$$

where we noted $\boldsymbol{\theta}=\left\{\mu_{n}, \sigma_{n}, \rho_{n}, n=0, \cdots, N\right\}$.

Note that

$$
-\ln p(\boldsymbol{x} \mid \boldsymbol{t}, \boldsymbol{\theta}, N)=\sum_{n=0}^{N}\left(l_{n} / 2\right) \ln (2 \pi)+\frac{1}{2} \sum_{n=0}^{N} \ln \left|\boldsymbol{\Sigma}_{n}\right|-\frac{1}{2} \sum_{n=0}^{N}\left(\boldsymbol{x}_{n}-\mu_{n} \mathbf{1}\right)^{\prime} \boldsymbol{\Sigma}_{n}^{-1}\left(\boldsymbol{x}_{n}-\mu_{n} \mathbf{1}\right)
$$

and when the data are i.i.d., $\left(\boldsymbol{\Sigma}_{n}=\sigma_{n} \boldsymbol{I}\right)$ this becomes

$$
-\ln p(\boldsymbol{x} \mid \boldsymbol{t}, \boldsymbol{\theta}, N)=(T / 2) \ln (2 \pi)+\sum_{n=0}^{N}\left(l_{n} / 2\right) \ln \sigma_{n}^{2}-\sum_{n=0}^{N} \frac{\left\|\left(\boldsymbol{x}_{n}-\mu_{n} \mathbf{1}\right)\right\|^{2}}{2 \sigma_{n}^{2}}
$$

Then, the inference problems we will be faced are the following: 
1. Infer on $\boldsymbol{\theta}$ given $\boldsymbol{x}$ and $\boldsymbol{t}$;

2. Infer on $\boldsymbol{t}$ given $\boldsymbol{x}$ and $\boldsymbol{\theta}$;

3. Infer on $\boldsymbol{t}$ and $\boldsymbol{\theta}$ given $\boldsymbol{x}$;

4. Infer on $\boldsymbol{\theta}$ given $\boldsymbol{x}$.

5. Infer on $\boldsymbol{t}$ given $\boldsymbol{x}$;

It is clear that the first problem is the easiest.

The classical maximum likelihood estimation (MLE) approach can handle only the first three problems by maximizing $p(\boldsymbol{x} \mid \boldsymbol{t}, \boldsymbol{\theta})$, respectively, with respect to $\boldsymbol{\theta}$, to $\boldsymbol{t}$ and jointly $(\widehat{\boldsymbol{t}}, \widehat{\boldsymbol{\theta}})$ :

- Estimating $\boldsymbol{\theta}$ given $\boldsymbol{x}$ and $\boldsymbol{t}: \quad \widehat{\boldsymbol{\theta}}=\arg \max _{\boldsymbol{\theta}}\{p(\boldsymbol{x} \mid \boldsymbol{t}, \boldsymbol{\theta})\}$

- Estimating $\boldsymbol{t}$ given $\boldsymbol{x}$ and $\boldsymbol{\theta}: \quad \widehat{\boldsymbol{t}}=\arg \max _{\boldsymbol{t}}\{p(\boldsymbol{x} \mid \boldsymbol{t}, \boldsymbol{\theta})\}$

- Estimating $\boldsymbol{t}$ and $\boldsymbol{\theta}$ given $\boldsymbol{x}: \quad(\widehat{\boldsymbol{t}}, \widehat{\boldsymbol{\theta}})=\arg \max _{(\boldsymbol{t}, \boldsymbol{\theta})}\{p(\boldsymbol{x} \mid \boldsymbol{t}, \boldsymbol{\theta})\}$

However, we must be careful to check the boundedness of the likelihood function before using any optimization algorithm. The optimization with respect to $\boldsymbol{\theta}$ when $\boldsymbol{t}$ is known can be done easily, but the optimization with respect to $\boldsymbol{t}$ is very hard and computationally costly.

The two last problems cannot be handled easily because they need to define the likelihood fuctions $p(\boldsymbol{x} \mid \boldsymbol{\theta})$ and $p(\boldsymbol{x} \mid \boldsymbol{t})$ which need integrations with respect to $\boldsymbol{t}$ or $\boldsymbol{\theta}$ of $p(\boldsymbol{x} \mid \boldsymbol{t}, \boldsymbol{\theta})$. There may not be possible to find analytical expressions for these integrals which may even not exist.

\section{BAYESIAN ESTIMATION OF THE CHANGE POINT TIME INSTANTS}

In Bayesian approach, one assigns prior probability laws on both $\boldsymbol{t}$ and $\boldsymbol{\theta}$ and use the posterior probability law $p(\boldsymbol{t}, \boldsymbol{\theta} \mid \boldsymbol{x})$ as a tool for doing any inference. Choosing a prior pdf for $\boldsymbol{t}$ is also usual in classical approach. A simple model is the following:

$$
t_{n}=t_{n-1}+\epsilon_{n} \quad \text { with } \quad \epsilon_{n} \sim \mathcal{P}(\lambda),
$$

where $\varepsilon_{n}$ are assumed iid end $\lambda$ is the a priori mean value of time intervals $\left(t_{n}-t_{n-1}\right)$. if $N$ is the number of changepoint we can take $\lambda=\frac{T}{N+1}$. With this modeling we have :

$$
\begin{aligned}
& p(\boldsymbol{t} \mid \lambda)=\prod_{n=1}^{N+1} \mathcal{P}\left(t_{n}-t_{n-1} \mid \lambda\right)=\prod_{n=1}^{N+1} e^{-\lambda} \frac{\lambda^{\left(t_{n}-t_{n-1}\right)}}{\left(t_{n}-t_{n-1}\right) !} \\
& \ln p(\boldsymbol{t} \mid \lambda)=-(N+1) \lambda+\ln (\lambda) \sum_{n=1}^{N+1}\left(t_{n}-t_{n-1}\right)-\sum_{n=1}^{N+1} \ln \left(\left(t_{n}-t_{n-1}\right) !\right)
\end{aligned}
$$

With this prior selection, we have

$$
p(\boldsymbol{x}, \boldsymbol{t} \mid \boldsymbol{\theta}, N)=p(\boldsymbol{x} \mid \boldsymbol{t}, \boldsymbol{\theta}, N) p(\boldsymbol{t} \mid \lambda, N)
$$


and

$$
p(\boldsymbol{t} \mid \boldsymbol{x}, \boldsymbol{\theta}, N) \propto p(\boldsymbol{x} \mid \boldsymbol{t}, \boldsymbol{\theta}, N) p(\boldsymbol{t} \mid \lambda, N)
$$

In Bayesian approach, one goes one step further with assigning prior probability laws to the hyperparameters $\boldsymbol{\theta}$, i.e., $p(\boldsymbol{\theta})$ and then one writes the joint a posteriori:

$$
p(\boldsymbol{t}, \boldsymbol{\theta} \mid \boldsymbol{x}, \lambda, N) \propto p(\boldsymbol{x} \mid \boldsymbol{t}, \boldsymbol{\theta}, N) p(\boldsymbol{t} \mid \lambda, N) p(\boldsymbol{\theta} \mid N)
$$

where here we noted $\boldsymbol{\theta}=\left\{\mu_{n}, \sigma_{n}^{2}, \rho_{n}, n=1, \cdots, N\right\}$.

To go further in details, we need to assign $p(\boldsymbol{\theta})$. The following is our selection:

$$
\begin{aligned}
p\left(\mu_{n}\right) & =\mathcal{N}\left(\mu_{0}, \sigma_{0}^{2}\right) \\
p\left(\sigma_{n}^{2}\right) & =\mathcal{I} \mathcal{G}\left(\alpha_{0}, \beta_{0}\right) \\
p\left(\rho_{n}\right) & =\mathcal{U}([0,1])
\end{aligned}
$$

which correspond mainely to the conjugate or reference priors.

Given all these, we propose the following Gibbs MCMC algorithm:

$$
\begin{aligned}
& \text { Iterate until convergency } \\
& \text { - sample } \boldsymbol{t} \quad \text { using } p(\boldsymbol{t} \mid \boldsymbol{x}, \boldsymbol{\theta}, N) \\
& \text { - sample } \theta_{n} \text { : } \\
& \begin{array}{lll}
\mu_{n} & \text { using } & p\left(\mu_{n} \mid \boldsymbol{x}, \boldsymbol{t}, N\right) \\
\sigma_{n}^{2} & \text { using } & p\left(\sigma_{n}^{2} \mid \boldsymbol{x}, \boldsymbol{t}, N\right) \\
\rho_{n} & \text { using } & p\left(\rho_{n} \mid \boldsymbol{x}, \boldsymbol{t}, N\right)
\end{array}
\end{aligned}
$$

\subsection{Sampling $\boldsymbol{t}$ using $p(\boldsymbol{t} \mid \boldsymbol{x}, \boldsymbol{\theta}, N)$}

P. Fearnhead showed ${ }^{11}$ that it is possible to perform perfect simulation of $p(\boldsymbol{t} \mid \boldsymbol{x}, \boldsymbol{\theta}, N)$ when we have assumed that segments of data separated by a changepoint $t_{n}$ are independant. This simulation can be obtained by a method based on recursion on the changepoints. An approximation of this method is possible to obtain an algorithm whose computational cost is linear in the number of observations. The main principle of this algorithm is to compute the following probabilities :

Let note $\boldsymbol{x}_{t: s}=[x(t), x(t+1), \ldots, x(s)]$, and

$$
\begin{aligned}
R(t, s \mid \lambda) & =p\left(\boldsymbol{x}_{t: s} \mid t, s \text { in the same segment, } \lambda\right) \\
Q(t \mid \lambda) & =p\left(\boldsymbol{x}_{t: s} \mid \text { changepoint at } t-1, \lambda\right), \quad Q(1)=p(\boldsymbol{x} \mid \lambda)
\end{aligned}
$$

Let also note $F(t \mid \lambda)$ the associated cumulative distribution function of the prior density $\mathcal{P}\left(t_{n}-\right.$ $\left.t_{n-1} \mid \lambda\right)$ which is defined by $(7)$.

We compute $R(t, s \mid \lambda)$ with the following relation :

$$
R(t, s) \mid \lambda)=\int p\left(\boldsymbol{x}_{t: s} \mid \boldsymbol{\theta}, \lambda\right) p(\boldsymbol{\theta}) d \boldsymbol{\theta}
$$


The computation of $Q(t \mid \lambda)$ can be done recursively by the following result : for $t=1, \ldots, T$,

$$
Q(t \mid \lambda)=\sum_{s=t}^{T-1} R(t, s \mid \lambda) Q(s+1 \mid \lambda) \mathcal{P}(s+1-t \mid \lambda)+R(t, T \mid \lambda)(1-F(T-t \mid \lambda)),
$$

This result is shown by P. Fearnhead ${ }^{11}$. And he also demonstrates that the posterior distribution of $t_{n}$ given $t_{n-1}$ is

$$
p\left(t_{n} \mid t_{n-1}, \boldsymbol{x}, \lambda\right)=\frac{R\left(t_{n-1}, t_{n} \mid \lambda\right) Q\left(t_{n}+1 \mid \lambda\right) \mathcal{P}\left(t_{n}-t_{n-1} \mid \lambda\right)}{Q\left(t_{n-1} \mid \lambda\right)}
$$

and the posterior distribution of no further changepoint is given by

$$
p\left(t_{n}=T \mid t_{n-1}, \boldsymbol{x}, \lambda\right)=\frac{R\left(t_{n-1}, T \mid \lambda\right)\left(1-F\left(T-t_{n-1}-1 \mid \lambda\right)\right)}{Q\left(t_{n-1} \mid \lambda\right)}
$$

\subsection{Sampling $\theta_{n}$ using $p\left(\theta_{n} \mid \boldsymbol{x}, \boldsymbol{t}, N\right)$}

We may note that, thanks to the conjugacy, we have:

$$
\begin{aligned}
& p\left(\mu_{n} \mid \boldsymbol{x}, \boldsymbol{t}\right)=\mathcal{N}\left(\widehat{\mu}_{n}, \widehat{\sigma}_{n}^{2}\right) \text { with }\left\{\begin{array}{l}
\widehat{\mu}_{n}=\widehat{\sigma}_{n}^{2}\left[\frac{\mu_{0}}{\sigma_{0}^{2}}+\mathbf{1}^{\prime} \boldsymbol{\Sigma}_{n}^{-1} \boldsymbol{x}_{n}\right] \\
\widehat{\sigma}_{n}^{2}=\left(\mathbf{1}^{\prime} \boldsymbol{\Sigma}_{n}^{-1} \mathbf{1}+\frac{1}{\sigma_{0}^{2}}\right)^{-1}
\end{array}\right. \\
& p\left(\sigma_{n}^{2} \mid \boldsymbol{x}, \boldsymbol{t}\right)=\mathcal{I} \mathcal{G}\left(\widehat{\alpha}_{n}, \widehat{\beta}_{n}\right) \text { with }\left\{\begin{array}{l}
\widehat{\alpha}_{n}=\alpha_{0}+\frac{l_{n}}{2} \\
\widehat{\beta}_{n}=\beta_{0}+\frac{1}{2}\left(\boldsymbol{x}_{n}-\mu_{n} \mathbf{1}\right)^{\prime} \boldsymbol{R}_{n}^{-1}\left(\boldsymbol{x}_{n}-\mu_{n} \mathbf{1}\right),
\end{array}\right.
\end{aligned}
$$

where $\boldsymbol{R}_{n}=$ Toeplitz $\left(\left[1, \rho_{n}, \rho_{n}^{2}, \cdots, \rho_{n}^{l_{n}}\right]\right)$. Then the simulation of these densities is quite simple.

$p\left(\rho_{n} \mid \boldsymbol{x}, \boldsymbol{t}\right)$ is not a classical law. Its expression is given by :

$$
\begin{aligned}
p\left(\rho_{n} \mid \boldsymbol{x}, \boldsymbol{t}, N\right) & =\prod_{n=0}^{N} p\left(\rho_{n} \mid \boldsymbol{x}_{n}, \boldsymbol{t}, N\right) \\
& \propto\left(\frac{1}{\sigma_{n}^{2}\left(1-\rho_{n}^{2}\right)}\right)^{\frac{l n}{2}} \exp \left\{-\frac{1}{2 \sigma_{n}^{2}\left(1-\rho_{n}^{2}\right)}\left(\boldsymbol{x}_{n}-\mu_{n} \mathbf{1}\right)^{\prime} \boldsymbol{R}_{n}^{-1}\left(\boldsymbol{x}_{n}-\mu_{n} \mathbf{1}\right)\right\} \\
& \propto\left(\frac{1}{\sigma_{n}^{2}\left(1-\rho_{n}^{2}\right)}\right)^{\frac{l n}{2}} \exp \left\{-\frac{1}{2 \sigma_{n}^{2}\left(1-\rho_{n}^{2}\right)} \sum_{l=1}^{l n}\left(x\left(t_{n}+l\right)-\rho_{n} x\left(t_{n}+l-1\right)-\left(1-\rho_{n}\right) \mu_{n}\right)^{2}\right\}
\end{aligned}
$$

Then we can not sample easily this density.

The solution we propose is to use, in this step, a Hastings-Metropolis algorithm for sampling this density. As an instrumental density we propose to use a Gaussian approximation of the posterior density, i.e., we estimate the mean $m_{\rho_{n}}$ and the variance $\sigma_{\rho_{n}}^{2}$ of $p\left(\rho_{n} \mid \boldsymbol{x}, \boldsymbol{t}, N\right)$ and we use a Gaussian law $\mathcal{N}\left(m_{\rho_{n}}, \sigma_{\rho_{n}}^{2}\right)$ to obtain a sample. This sample is accepted or rejected 
following $p\left(\rho_{n} \mid \boldsymbol{x}, \boldsymbol{t}, N\right)$. In practice we compute $m_{\rho_{n}}$ and $\sigma_{\rho_{n}}^{2}$ calculating by approximation of their definition :

$$
\begin{aligned}
m_{\rho_{n}} & \longrightarrow \int_{0}^{1} \rho_{n} \quad p\left(\rho_{n} \mid \boldsymbol{x}, \boldsymbol{t}, N\right) \\
\sigma_{\rho_{n}}^{2} & \longrightarrow \int_{0}^{1} \rho_{n}^{2} \quad p\left(\rho_{n} \mid \boldsymbol{x}, \boldsymbol{t}, N\right)-m_{\rho_{n}}^{2}
\end{aligned}
$$




\section{OTHER FORMULATIONS}

Other formulation can also exist. We introduce two sets of hidden variables

$$
\boldsymbol{z}=\left[z\left(t_{0}\right), \cdots, z\left(t_{0}+T\right)\right]^{\prime} \text { and } \boldsymbol{q}=\left[q\left(t_{0}\right), \cdots, q\left(t_{0}+T\right)\right]^{\prime}
$$

where

$$
q(t)=\left\{\begin{array}{ll}
1 & \text { if } z(t) \neq z(t-1) \\
0 & \text { elsewhere }
\end{array}=\left\{\begin{array}{ll}
1 & \text { if } t=t_{n}, n=0, \cdots, N \\
0 & \text { elsewhere }
\end{array} .\right.\right.
$$

and where $z(t)$ takes an integer value $k$ in each segment : $k=1, \ldots, N+1$. With these two related hidden variables, we can propose two other modeling to be used in change point analysis. For example, $\boldsymbol{q}$ can be modeled by a Bernouilli process

$$
P(\boldsymbol{Q}=\boldsymbol{q})=\lambda^{\sum_{j} q_{j}}(1-\lambda)^{\sum_{j}\left(1-q_{j}\right)}=\lambda^{\sum_{j} q_{j}}(1-\lambda)^{N-\sum_{j} q_{j}}
$$

and $\boldsymbol{z}$ can be modeled by a Mrkov chain, i.e., $\{z(t), t=1, \cdots, T\}$ forms a Markov chain:

$$
\begin{aligned}
& P(z(t)=k)=p_{k}, \quad k=1, \cdots, K \\
& P(z(t)=k \mid z(t-1)=l)=p_{k l}, \quad \text { with } \quad \sum_{k} p_{k l}=1 .
\end{aligned}
$$

These two models are related. In the first one, $\lambda$ plays the role of the mean value of the segment lengths and in the second $p_{k}$ and $p_{k l}$ give more precise control of the segment lengths. In the multivariate case, or more precisely in bivariate case (image processing), $\boldsymbol{q}$ may represent the contours and $\boldsymbol{z}$ the labels for the regions in the image. Then, we may also give a Markov model for them. For example, if we note by $r \in \mathcal{S}$ the position of a pixel, $\mathcal{S}$ the set of pixels positions and by $\mathcal{V}(r)$ the set of pixels in the neighorhood of the pixel position $r$, we may use an Ising model for $\boldsymbol{q}$

$$
P(\boldsymbol{Q}=\boldsymbol{q}) \propto \exp \left\{-\rho \sum_{r \in \mathcal{S}} \sum_{s \in \mathcal{V}(r)} \delta(z(r)-z(s))\right\}
$$

or a Potts model for $\boldsymbol{z}$ :

$$
P(\boldsymbol{z}) \propto \exp \left\{-\rho \sum_{r \in \mathcal{S}} \sum_{s \in \mathcal{V}(r)} \delta(z(r)-z(s))\right\}
$$

where rho in the first controls the mean lengths of the contours in the image and in the second the mean surface of the regions in the image. Other more complexe modelings are also possible.

With these auxiliary variables, we can write

$$
p(\boldsymbol{x} \mid \boldsymbol{z}, \boldsymbol{\theta})=\sum_{n=1}^{N} P\left(z_{j}=n\right) \mathcal{N}\left(\mu_{n} \mathbf{1}, \boldsymbol{\Sigma}_{n}\right)=\sum_{n=1}^{N} p_{k} \mathcal{N}\left(\mu_{n} \mathbf{1}, \boldsymbol{\Sigma}_{n}\right)
$$

if we choose $K=N$. Here, $\boldsymbol{\theta}=\left\{N,\left\{\mu_{n}, \sigma_{n}, p_{n}, n=1, \cdots, N\right\},\left(p_{k l}, k, l=1, \cdots, N\right)\right\}$ and the model is a mixture of Gaussians.

We can again assign appropriate prior law on $\boldsymbol{\theta}$ and give the expression of $p(\boldsymbol{z}, \boldsymbol{\theta} \mid \boldsymbol{x})$ and do any inference on $\boldsymbol{z}, \boldsymbol{\theta}$. 
Finally, we can also use $\boldsymbol{q}$ as the auxiliary variable and write

$$
\begin{aligned}
p(\boldsymbol{x} \mid \boldsymbol{q}, \boldsymbol{\theta}) & =(2 \pi)^{-N / 2}\left(\prod_{n=1}^{N} 1 / \sigma_{n}\right) \exp \left\{-\frac{1}{2 \sigma_{n}^{2}} \sum_{n=1}^{N}\left(x\left(t_{n}\right)-\mu_{n}\right)^{2}\right\} \\
& +(2 \pi)^{-(T-N) / 2}\left(\prod_{n=1}^{N} 1 / \sigma_{n}^{\left(l_{n}-1\right)}\right) \exp \left\{-\frac{1}{2 \sigma_{n}^{2}} \sum_{j=1}^{T}\left(1-q_{j}\right)\left(x_{j}-x_{j-1}\right)^{2}\right\} \\
& =(2 \pi)^{-T / 2}\left(\prod_{n=1}^{N} 1 / \sigma_{n}^{\left(l_{n}\right)}\right) \exp \left\{-\frac{1}{2 \sigma_{n}^{2}} \sum_{j=1}^{T}\left[\left(1-q_{j}\right)\left(x_{j}-x_{j-1}\right)^{2}+q_{j}\left(x_{j}-\mu_{n}\right)\right]\right\}
\end{aligned}
$$

and again assign appropriate prior law on $\boldsymbol{\theta}$ and give the expression of $p(\boldsymbol{q}, \boldsymbol{\theta} \mid \boldsymbol{x})$ and do any inference on $\boldsymbol{q}, \boldsymbol{\theta}$. We are still working on using these auxiliary hidden variables particularly for applications in data fusion in image processing and we will report on these works very soon. 


\section{SIMULATION RESULTS}

To test the feasability and to mesaure the performances of the proposed algorithms, we generated a few simple cases corresponding to only changes of one of the three parameters $\mu_{n}, \sigma_{n}^{2}$ and $\rho_{n}$. In each case we present the data, the histogram of the a posteriori samples of $\boldsymbol{t}$ during the first and the last iterations of the MCMC algorithm. For each case we also give the value of the

parameters used to simulate the data, the estimated values when the changepoints are known and the estimated values by the proposed method. 


\subsection{Change of the means}

We can see in figure 2 that we obtain precise results on the position of the changepoints. In the case of change of means, the algorithm is very fast to converge to the good solution. In fact it needs only few iterations (about 5). The main cause of this results is the importance of the means in the likelihood $p(\boldsymbol{x} \mid \boldsymbol{t}, \boldsymbol{\theta}, N)$.

We can also see in table 1 that the estimations of the means are very precise, particularly when the size of the segment is long.

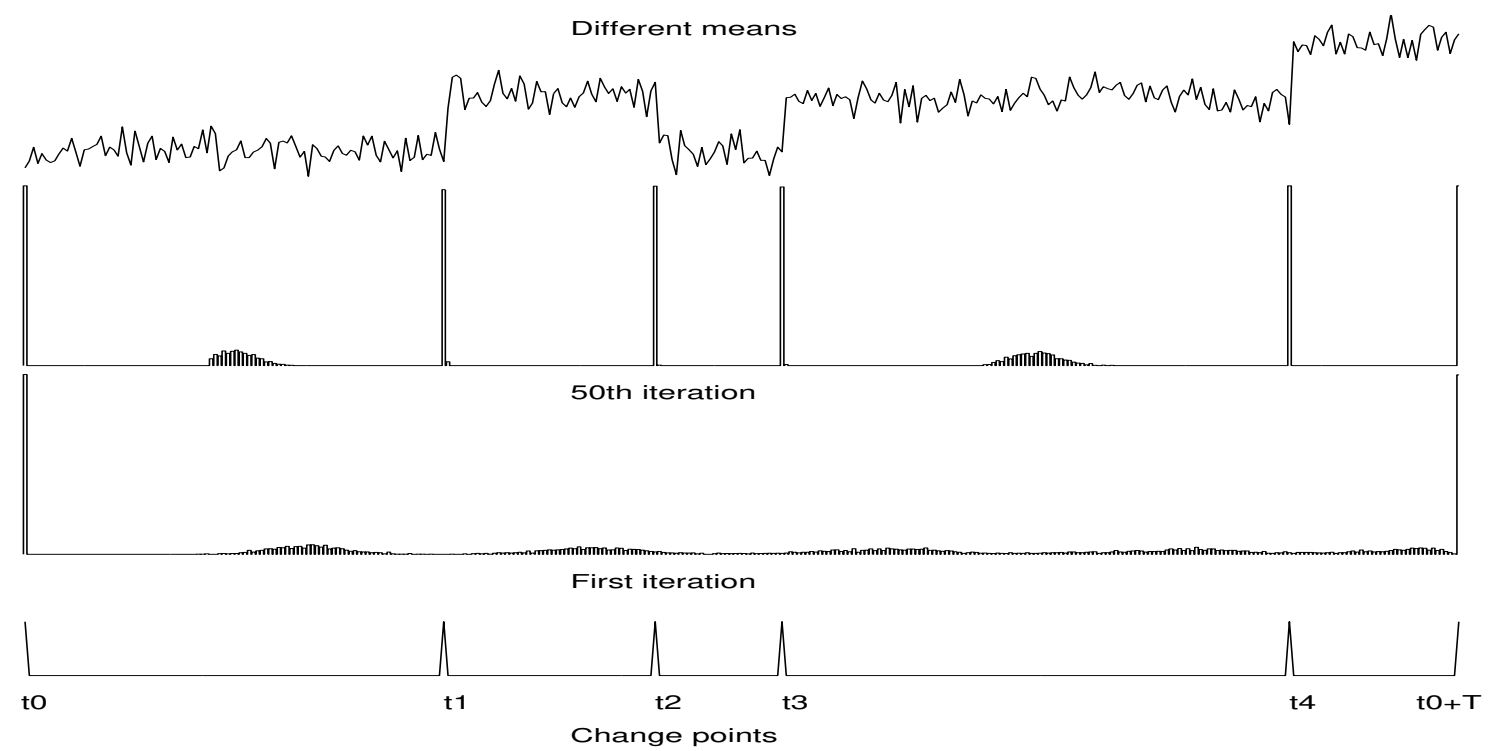

Figure 2. Change in the means. up to down : simulated data, histogram in the 50th iteration, histogram in the first iteration, real position of the changepoints.

\begin{tabular}{|c|c|c|}
\hline $\mathrm{m}$ & $\hat{m} \mid \boldsymbol{x}, \boldsymbol{t}$ & $\hat{m} \mid \boldsymbol{x}$ \\
\hline \hline 1.5 & 1.4966 & 1.4969 \\
1.7 & 1.7084 & 1.7013 \\
1.5 & 1.4912 & 1.5015 \\
1.7 & 1.6940 & 1.6929 \\
1.9 & 1.9012 & 1.8915 \\
\hline
\end{tabular}

Table 1. Estimated value of the means 


\subsection{Change in the variances}

We can see in figure 3 that we have again good results on the position of the changepoints. However, for little difference of variances, the algorithm give an uncertainty on the exact position of the changepoint. This can be justified by the fact that the simulated data give itself this uncertainty.

In table 2 we can see again good estimations on the variances on each segments.

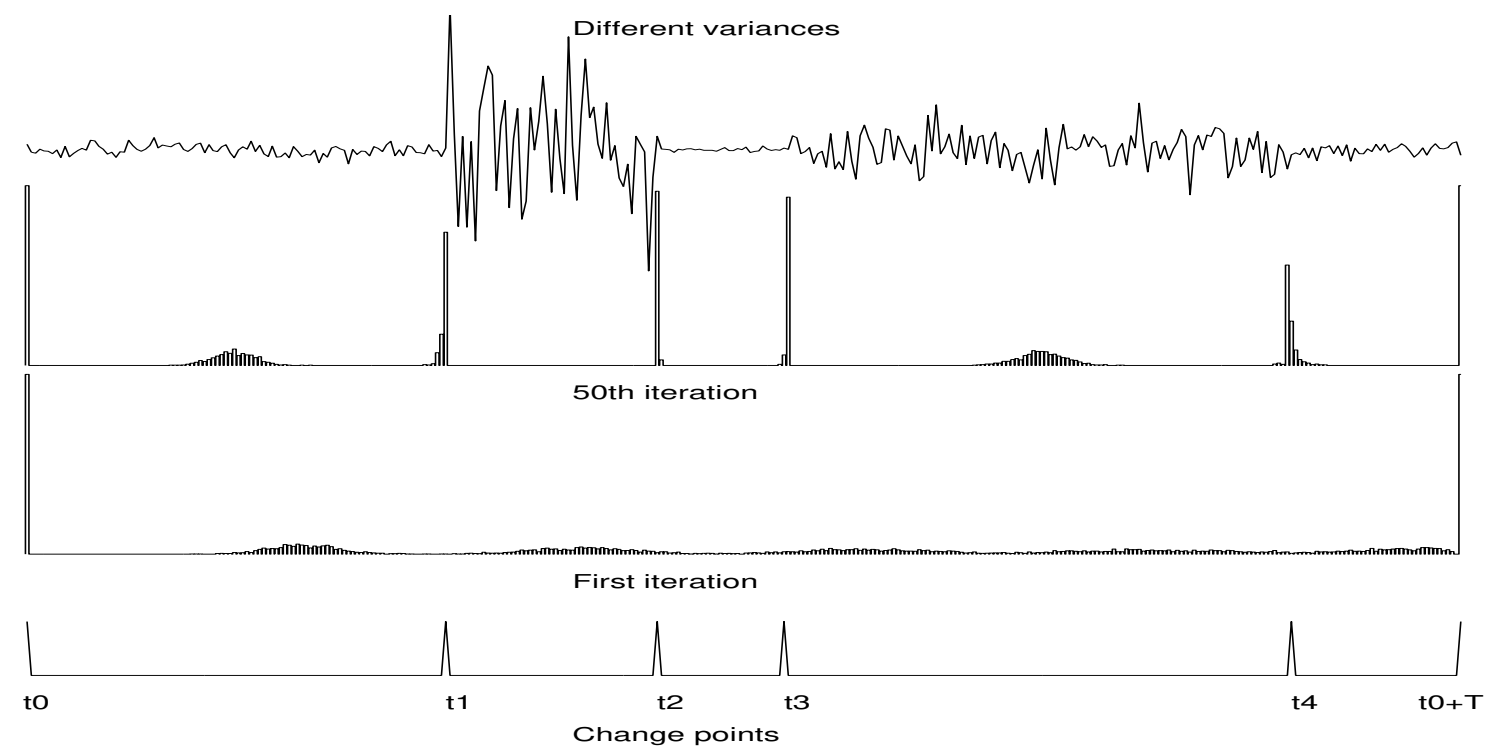

Figure 3. Change in the variances. up to down : simulated data, histogram in the 50th iteration, histogram in the first iteration, real position of the changepoints.

\begin{tabular}{|c|c|c|}
\hline$\sigma^{2}$ & $\hat{\sigma}^{2} \mid \boldsymbol{x}, \boldsymbol{t}$ & $\hat{\sigma}^{2} \mid \boldsymbol{x}$ \\
\hline \hline 0.01 & 0.0083 & 0.0081 \\
1 & 0.9918 & 0.9598 \\
0.001 & 0.0007 & 0.0026 \\
0.1 & 0.0945 & 0.0940 \\
0.01 & 0.0079 & 0.0107 \\
\hline
\end{tabular}

Table 2. Estimated value of the variances 


\subsection{Change in the correlation coefficient}

The results showed in figure 4 are worse than in the two first cases. The position of the changepoints are less precise, and we can see that another changepoint appears. This affects the estimation of the correlation coefficient in the third segment because the algorithm alternates between two positions of changepoint. This problem can be justified by the fact that a value of the correlation coefficient near 1 implies locally a change of the mean, which can be considered by the algorithm as a changepoint. Also this problem appears when the size of the segments are far from the a priori size $\lambda$.

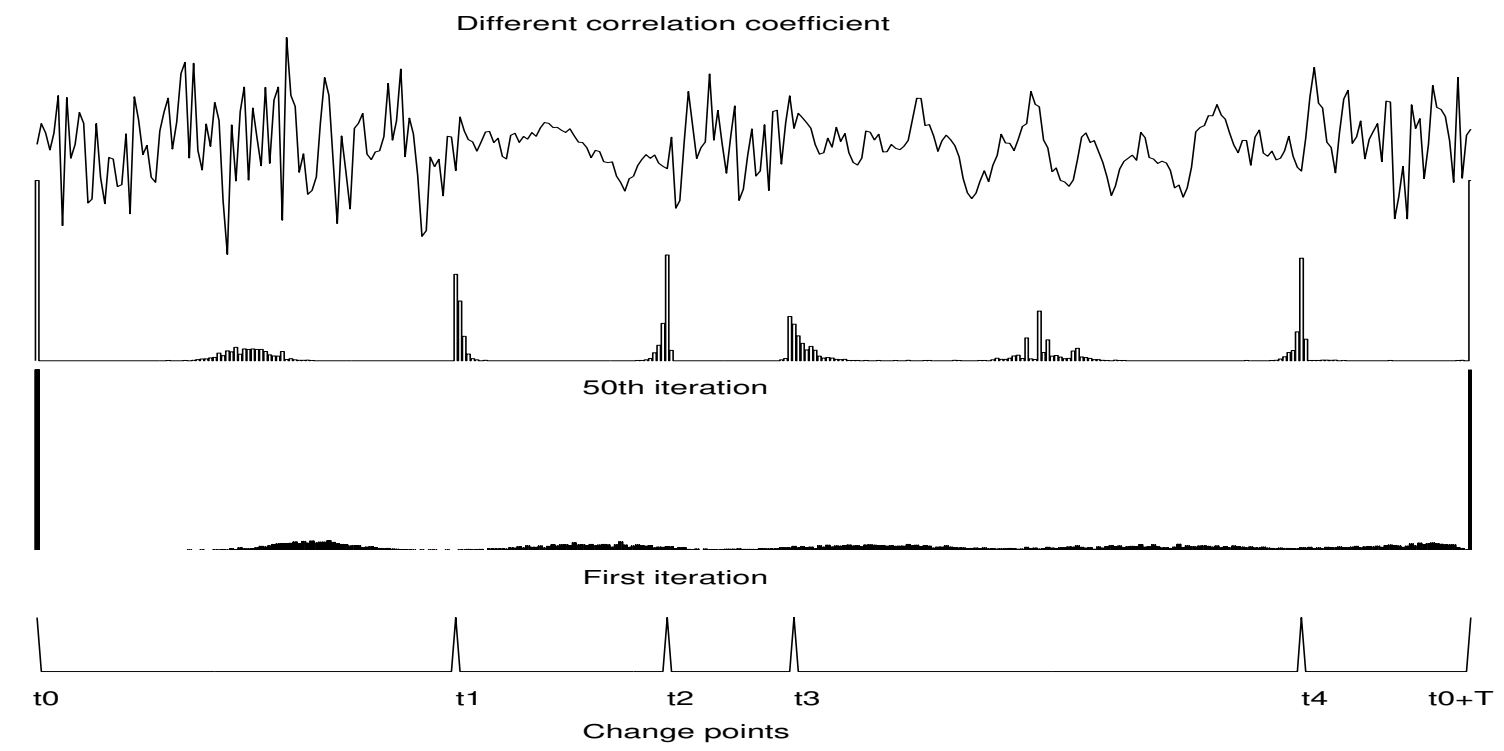

Figure 4. Change in the correlation coefficient. up to down : simulated data, histogram in the 50th iteration, histogram in the first iteration, real position of the changepoints.

\begin{tabular}{|c|c|}
\hline$a$ & $\hat{a} \mid \boldsymbol{x}$ \\
\hline \hline 0 & 0.0988 \\
0.9 & 0.7875 \\
0.1 & 0.3737 \\
0.8 & 0.8071 \\
0.2 & 0.1710 \\
\hline
\end{tabular}

Table 3. Estimated vaue of the correlation coefficients 


\subsection{Influence of the prior law}

In this section we study the influence of the a priori on $\lambda$, i.e., the size of the segments. In the following we fix the number of changepoints as before and we change the a priori size of the segments by $\lambda_{0}=\frac{\lambda}{2}$ and $\lambda_{1}=2 \lambda$. We apply then our algorithm on the change of the correlation coefficient.

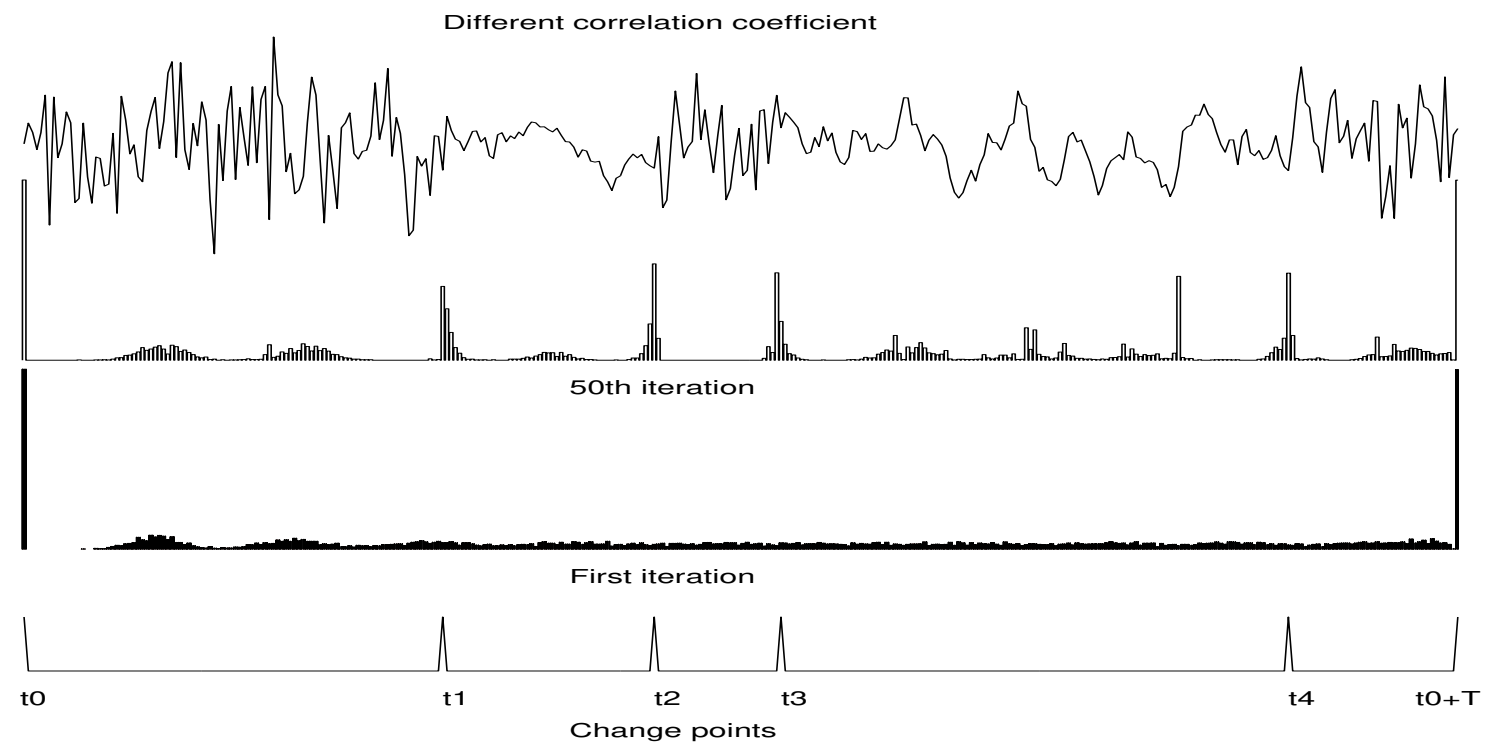

Figure 5. Different correlation coefficient with $\lambda_{0}=\frac{1}{2} \frac{T}{N+1}$. up to down : simulated data, histogram in the 50th iteration, histogram in the first iteration, real position of the changepoints.

In figure 5, we can see that the algorithm has detected other changepoints, forming segments whose size is near $\lambda_{0}$. This result shows the importance of the a priori when the data are not enough significant. We can also see this conclusion in figure 6 where only three changepoints are detected, forming segments whose size is again near $\lambda_{1}$. We can also remark that fixing $a$ priori a size $\lambda$ comes down to fix the number of changepoints. Our algorithm give then good results for instance if we have a good a priori on the number of changepoints.

\section{CONCLUSIONS}

\section{REFERENCES}

1. M. Basseville, "Detecting changes in signals and systems - a survey," Automatica, vol. 24, no. 3, pp. 309-326, 1988.

2. M. Wax, "Detection and localization of multiple sources via the stochastic signals model," IEEE Transactions on Signal Processing, vol. 39, pp. 2450-2456, November 1991.

3. J. J. Kormylo and J. M. Mendel, "Maximum-likelihood detection and estimation of BernoulliGaussian processes," IEEE Transactions on Information Theory, vol. 28, pp. 482-488, 1982. 


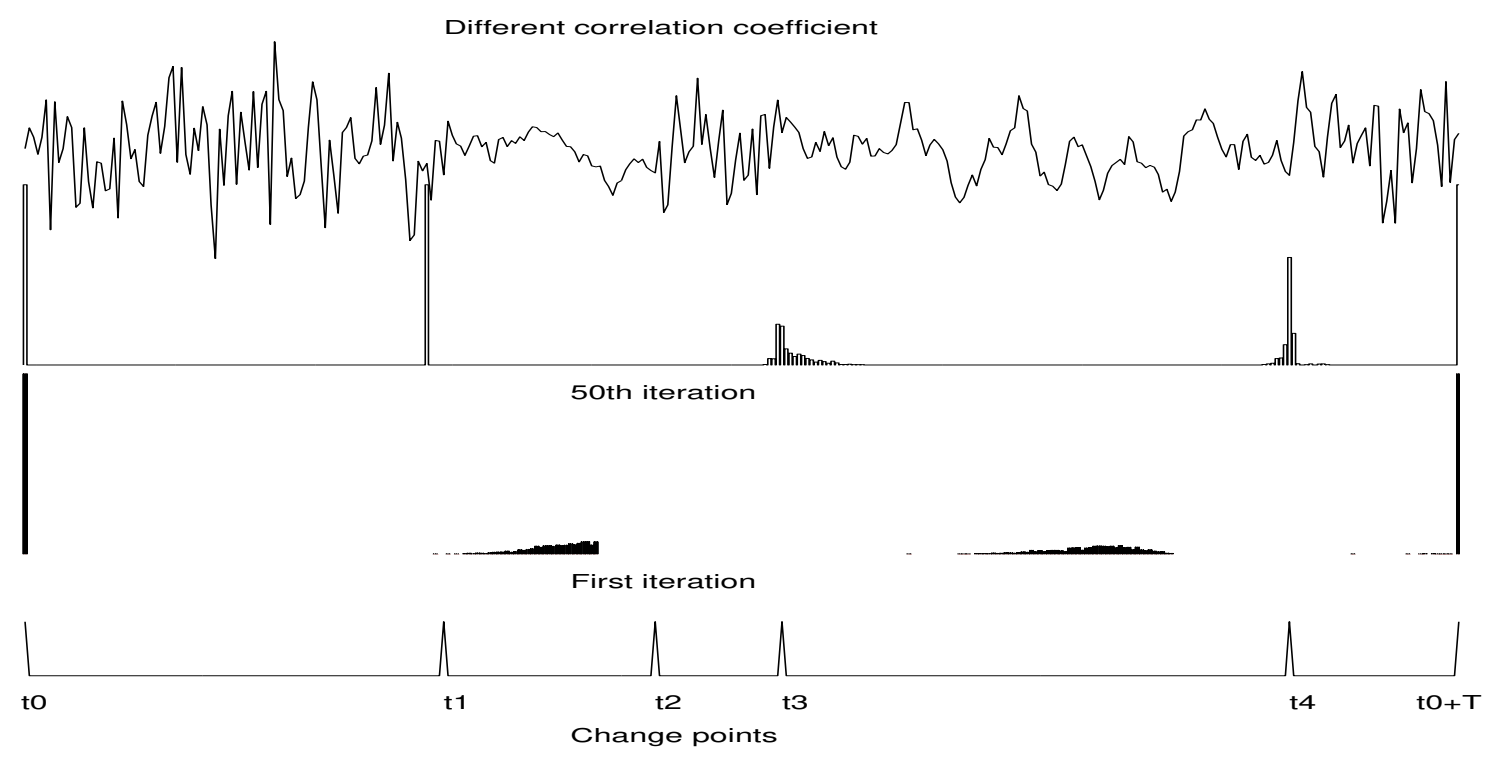

Figure 6. Different correlation coefficient with $\lambda_{1}=2 \frac{T}{N+1}$. up to down : simulated data, histogram in the 50th iteration, histogram in the first iteration, real position of the changepoints.

4. C. Y. Chi, J. Goustias, and J. M. Mendel, "A fast maximum-likelihood estimation and detection algorithm for Bernoulli-Gaussian processes," in Proceedings of the International Conference on Acoustic, Speech and Signal Processing, (Tampa, FL), pp. 1297-1300, April 1985.

5. J. K. Goutsias and J. M. Mendel, "Optimal simultaneous detection and estimation of filtered discrete semi-Markov chains," IEEE Transactions on Information Theory, vol. 34, pp. 551-568, 1988.

6. J. J. Oliver, R. A. Baxter, and C. S. Wallace, "Unsupervised Learning using MML," in $M a$ chine Learning: Proceedings of the Thirteenth International Conference (ICML 96), pp. 364-372, Morgan Kaufmann Publishers, 1996.

7. Y. Liu, W. Wang, and V. Kilic, "Intensity particle flow smc-phd filter for audio speaker tracking," arXiv preprint arXiv:1812.01570, 2018.

8. J. P. Hughes, P. Guttorp, and S. P. Charles, "A non-homogeneous hidden Markov model for precipitation occurrence," Applied Statistics, vol. 48, no. 1, pp. 15-30, 1999.

9. L. J. Fitzgibbon, L. , and D. L. Dowe, "Minimum message length grouping of ordered data," in Algorithmic Learning Theory, 11th International Conference, ALT 2000, Sydney, Australia, December 2000, Proceedings, vol. 1968, pp. 56-70, Springer, Berlin, 2000.

10. L. Fitzgibbon, D. L. Dowe, and L. Allison, "Change-point estimation using new minimum message length approximations," in Proceedings of the Seventh Pacific Rim International Conference on Artificial Intelligence (PRICAI-2002) (M. Ishizuka and A. Sattar, eds.), vol. 2417 of LNAI, (Berlin), pp. 244-254, Japanese Society for Artificial Intelligence (JSAI), Springer-Verlag, August 2002.

11. P. Fearnhead, "Exact and efficient bayesian inference for multiple changepoint problems," tech. rep., Department of math. and stat., Lancaster university. 